\title{
Dosimetric comparison of intensity-modulated proton radiotherapy versus intensity-modulated photon-based radiotherapy for breast cancer
}

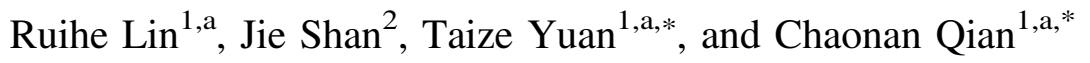 \\ 1 Department of Radiation Oncology, Guangzhou Concord Cancer Center, No. 9 Ciji Road, 510555 Guangzhou, PR China \\ 2 Department of Radiation Oncology, Mayo Clinic Arizona, Scottsdale, Arizona 85259, USA
}

Received 19 January 2021, Accepted 29 June 2021, Published online 20 August 2021

\begin{abstract}
Purpose: This study aims to compare the dosimetric differences in intensity-modulated proton therapy (IMPT) using pencil beam scanning technology and intensity-modulated photon-based radiotherapy (IMRT) in hypofractionated whole-breast irradiation (HF-WBI) and find out the more beneficial technique.

Methods and Materials: Eight breast cancer (BC) patients with pathological stage T1 2N0M0 were immobilized and underwent 4D-CT scanning used deep inspiration breath-hold (DIBH) technology. The IMPT and IMRT plans were designed for each patient. The IMPT plans used two en-face beam angles. IMRT plans were designed using the field in field technique. The optimization constraints of the two types of plans were identical. Prescription dose and regimen was $40.05 \mathrm{~Gy}$ (relative biological effect [RBE])/15 fx with a $10 \mathrm{~Gy}(\mathrm{RBE}) / 5 \mathrm{fx}$ boost, five fractions a week. A dose of $95 \%$ of the target volume should not be less than the prescribed dose. The target coverage was evaluated using D1, D2, D50, D95, D98, and D99. The target dose distribution and conformity were evaluated using the Conformity index (CI) and the homogeneity index (HI). The Organs at risk (OARs) were evaluated using mean dose (Dmean) and maximum dose (Dmax). Ipsilateral Lung and Contralateral Lung were evaluated additionally using V5, V10, V20, V30.

Results: The mean dose (Dmean) of the Heart $(P=0.012)$, Ipsilateral Lung $(P=0.036)$, Contralateral Lung $(P=0.012)$, and Spinal Cord $(P=0.012)$ were significantly reduced in IMPT plans. The IMPT also showed a tendency to reduce the V20 $(P=0.05)$ and V30 $(P=0.05)$ of the Ipsilateral Lung. But there was no significant difference in target coverage, homogeneity, and conformity between the IMRT and IMPT plans.

Conclusion: Compared to IMRT, the IMPT using pencil beam scanning technology can spare OARs without compromising target coverage in $\mathrm{BC}$ patients undergoing HF-WBI, which potentially reduce the incidence of radiation-related adverse effects and thus may positively impact long-term survival.
\end{abstract}

Key words: Whole breast irradiation, Proton therapy, Dosimetry comparison, Pencil beam scanning.

\section{Introduction}

Breast cancer (BC) is the most common cancer in women. Since the 21 st century, breast-conserving surgery (BCS) combined with radiotherapy has replaced mastectomy as the standard treatment for early-stage BC [1]. Adjuvant radiotherapy after BCS can halve the rate at which the disease recurs and reduce breast cancer death rate by about a sixth [2]. Several trials [3-9] published in recent years have shown various regimen of hypo-fractionated whole-breast irradiation (HF-WBI) is safe and effective as to the conventional fractionated whole-breast irradiation (CF-WBI) in early-stage BC patients. However, radiation-induced toxicity to normal tissue, particularly ischemic heart disease and secondary primary tumor in irradiation fields,

\footnotetext{
*Corresponding authors: chaonan.qian@ccm.cn; taize.yuan@ccm.cn
}

${ }^{\text {a }}$ Contributed equally as co-first authors. is a key factor negatively affecting both survival and quality of life [10-14]. Therefore, sparing normal tissue without compromising target coverage is crucial to prevent radiation-induced toxicity and thus improve survival and quality of life. Due to the Bragg peak, proton therapy can get a rapid fall-off of the dose at the distal end of the target volume, which makes proton therapy a good candidate. A recent meta-analysis [15] has shown that proton therapy could reduce mean heart dose in breast cancer irradiation, and decrease late cardio-vascular toxicity potentially. A prospective study [16] proved that proton therapy for regional nodal irradiation (RNI) after mastectomy or BCS reduced cardiac dose, lung V5, and V20 significantly compared with photons. Patel et al. [17] showed that for leftsided post-mastectomy radiation, proton therapy can improve 
homogeneity and cardiopulmonary sparingly compared to partially wide tangent photon with DIBH. In most of the previous studies, proton therapy was performed in conventional technique by using passively scattered scanning, which was inconsistent with the current radiotherapy pattern. Herein we conducted this study to compare the dosimetric differences in intensity-modulated proton (IMPT) using pencil beam scanning (PBS) technology and intensity-modulated photon-based radiotherapy (IMRT) in HF-WBI and find a more beneficial technique.

\section{Methods and materials}

\section{Patients}

Eight BC patients with pathological stage T1 2N0M0 underwent HF-WBI with a tumor-bed boost at the Mayo Clinic Arizona from March to November 2020 were enrolled, with a median age of 59.5 years (33-79 years old). Among them, five cases were left-sided disease and three cases were right-sided disease. All of them completed standardized BCS and medical treatment prior to radiotherapy. The pathology was all diagnosed as invasive ductal carcinoma with a negative margin. All eight patients had Karnofsky performance status (KPS) score $\geq 90$ before radiotherapy.

\section{Simulation}

Patients were immobilized in the supine position using a carbon fiber breast bracket with both hands raised. Lead wires were used to mark surgical scar, whole breast, midline of sternum, and the midline of axilla. All patients underwent 4D-CT scanning taken from mandible to upper abdomen without contrast at $2 \mathrm{~mm}$ slice. The deep inspiration breath-hold (DIBH) technology was used in all eight patients.

\section{Target delineation}

Target volumes were delineated in the Eclipse 15.0 system. The tumor bed (Lumpectomy) was operation cavity after resection including the excision cavity volume observed on CT, architectural distortion, lumpectomy scar, seroma, and extent of surgical clips. The CTV_Lumpectomy automatically included a 3D automatic $10 \mathrm{~mm}$ margin around the Lumpectomy, correcting manually to take into account anatomy and natural barriers. CTV_Breast was the visible breast tissue with a $5 \mathrm{~mm}$ contraction from the skin, including CTV_Lumpectomy. The range of clinical markers was taken for reference when delineated CTV_Lumpectomy. All the PTVs were obtained by a $5 \mathrm{~mm}$ expansion in 3D directions from relative CTVs. The organs at risk (OARs) were delineated on the planning CT included the Ipsilateral Lung, Contralateral Lung, Heart, and Spinal Cord according to the Radiation Therapy Oncology Group atlas [18]. The same contours were used for the IMPT and IMRT plans. For left-sided cases, all the contours were transferred to the DIBH scan using deformable registration and then appropriately corrected.

\section{Treatment planning}

A total of 16 plans ( 8 for IMRT and 8 for IMPT) were generated and analyzed. The IMRT plans and the IMPT plans were completed by two experienced physicists respectively. The treatment plans for IMRT and IMPT were both generated using the Treatment Planning System (TPS) of Eclipse (Varian Medical System, Inc., California, USA). The IMPT plans used two en-face beam angles and the IMPT with pencil beam weights was obtained through robust single-field optimization (SFO) technique. The IMRT plans were designed using the field in field technique. We chose an anisotropic analytical algorithm (AAA) for IMRT and a proton convolution superposition (PCS) algorithm for IMPT. Due to the limitations of the retrospective study, we had been unable to see the previous specific optimization constraints in the Eclipse system when we started this study. The IMPT and IMRT plans were both created with the best effort to meet the dose constraints shown in Table 1, although the optimization constraints used might be slightly different.

\section{Dose prescription}

The prescribed dose of the present study referred to the regimen of START B [4]: 40.05 Gy in 2.67-Gy fractions of relative biological effect (RBE) to PTV_Breast with a $10 \mathrm{~Gy}$ in 2-Gy fractions (RBE) additional boost to PTV_Lumpectomy. The RBE value was defined as 1.1 in the present study.

\section{Plan evaluation}

Target coverage should meet the criteria that the dose of $95 \%$ of the target volume $\geq$ prescribed dose. Doses to the OARs should be kept to a minimum. The following dosevolume parameters were collected to evaluate the coverage of target volumes: D1, D2, D50, D95, D98, D99. According to the ICRU83 report [19], refer to D2, D98 as Dmax (maximum dose) and Dmin (minimum dose) respectively. Homogeneity index (HI) was used as the standard formula to evaluate the homogeneity of the PTV, defined as D95/D5. Conformity index (CI) is defined as a ratio between V95\% and the target volume. All the OARs were evaluated using Dmean and Dmax. Ipsilateral Lung and Contralateral Lung were evaluated additionally using V5, V10, V20, V30.

\section{Statistical methods}

The paired dose-volume parameters should be normalized according to the data of D50 of the PTV_Lumpectomy. Our statistical analysis was performed on the normalized data.

The clinical data of eight patients included in the study were analyzed using the SPSS 23.0 software. The paired differences between IMPT and IMRT methods for each dose-volume parameter were assessed with the Wilcoxon signed-rank sum test. A two-sided $P$ value $<0.05$ was considered statistically significant. 
Table 1. Dose constrains.

\begin{tabular}{|c|c|c|c|}
\hline Structure & Volume type & Index & Target value \\
\hline CTV_Lumpectomy & Target & $\mathrm{D} 90 \%>$ & $90(\%$ of dose $)$ \\
\hline CTV_Lumpectomy & Target & $\mathrm{D} 95 \%>$ & $95(\%$ of dose $)$ \\
\hline CTV_Lumpectomy & Target & $\mathrm{V} 110 \%<$ & $0.01 \mathrm{cc}$ \\
\hline CTV_Lumpectomy & Target & $\mathrm{V} 115 \%<$ & $0.01 \mathrm{cc}$ \\
\hline CTV_Breast & Target & $\mathrm{D} 90 \%>$ & $90(\%$ of dose $)$ \\
\hline CTV_Breast & Target & $\mathrm{D} 95 \%>$ & $95(\%$ of dose $)$ \\
\hline Lung_I & OAR & $\mathrm{V} 40 \%<$ & $15 \sim 20$ ( $\%$ of volume $)$ \\
\hline Lung_C & OAR & V5 Gy < & $10 \sim 15$ ( $\%$ of volume $)$ \\
\hline Lung_C & OAR & $\mathrm{V} 10 \%<$ & $10 \sim 15$ ( $\%$ of volume $)$ \\
\hline Thyroid & OAR & D1 $\mathrm{cc}<$ & $90 \sim 100(\%$ of dose $)$ \\
\hline Cord & OAR & D0.01 $\mathrm{cc}<$ & $72(\%$ of dose $)$ \\
\hline Esophagus & OAR & D0.01 $\mathrm{cc}<$ & $32 \sim 36 \mathrm{~Gy}$ \\
\hline Esophagus & OAR & $\mathrm{D} 1 \mathrm{cc}<$ & $29 \sim 34 \mathrm{~Gy}$ \\
\hline Esophagus & OAR & D0.01 $\mathrm{cc}<$ & $72 \sim 90(\%$ of dose $)$ \\
\hline Hum_Head_I & OAR & D0.01 $\mathrm{cc}<$ & $35 \sim 43$ Gy \\
\hline Hum_Head_I & OAR & D1 $\mathrm{cc}<$ & $25 \sim 38 \mathrm{~Gy}$ \\
\hline Hum_Head_I & OAR & D0.01 $\mathrm{cc}<$ & $70 \sim 86(\%$ of dose $)$ \\
\hline Hum_Head_C & OAR & D0.01 $\mathrm{cc}<$ & $35 \sim 43 \mathrm{~Gy}$ \\
\hline Hum_Head_C & OAR & D1 $\mathrm{cc}<$ & $25 \sim 38 \mathrm{~Gy}$ \\
\hline Hum_Head_C & OAR & D0.01 $\mathrm{cc}<$ & $70 \sim 86(\%$ of dose $)$ \\
\hline Heart & OAR & Dmean $<$ & $1.5 \sim 3(\%$ of dose $)$ \\
\hline Heart & OAR & V50 Gy < & 1 (\% of volume) \\
\hline Brachia_Plex_I & OAR & D0.01 $\mathrm{cc}<$ & $102 \sim 108(\%$ of dose $)$ \\
\hline Skin & OAR & $\mathrm{D} 1 \mathrm{cc}<$ & $86 \sim 96(\%$ of dose $)$ \\
\hline LAD & OAR & D0.01 $\mathrm{cc}<$ & $6 \sim 30(\%$ of dose $)$ \\
\hline
\end{tabular}

D90\%, D95\%, D1 cc, D0.01 cc: dose receiving $\geq 90 \%$ relative volume, 95\% relative volume, 1 cc, 0.1 cc, respectively, Dmean: mean dose, V110\%, V115\%, V40\%, V10\%, V5 Gy, V50 Gy: volume receiving $\geq 110 \%$ prescription dose, 115\% prescription dose, $40 \%$ prescription dose, $10 \%$ prescription dose, 5 Gy, 50 Gy, respectively.

\section{Results}

\section{Target coverage}

A dosimetric comparison of PTV_Lumpectomy between IMPT and IMRT plans is presented in Table 2. The dose coverage criteria were achieved with both IMRT and IMPT plans for the PTV_Lumpectomy. The median dose of PTV_Lumpectomy D95 in IMPT and IMRT were $5388.678 \mathrm{cGy}$ and $5396.898 \mathrm{cGy}$, respectively, and the difference was not statistically significant $(P=0.889)$. The median dose of PTV Lumpectomy D1 in IMPT is $5481.351 \mathrm{cGy}$, which is similar to that in IMRT (5479.506 cGy) $(P=0.093)$. Similarly, differences in other dosimetric parameters of PTV_ Lumpectomy between the two groups were also not statistically significant.

\section{Organs at risk}

Table 3 summarized the dosimetric comparison between IMPT and IMRT plan for all the OARs and Video 1 showed that the OARs can benefit from IMPT plans. The median Heart Dmean was 9.524 cGy (RBE) (range, $0.033 \sim 42.380$ cGy [RBE]) with IMPT and $205.219 \mathrm{cGy}$ (range, $13.059 \sim 2668.997 \mathrm{cGy}$ ) with IMRT, an almost 20-fold increase $(P=0.012)$. For Ipsilateral Lung, the median Dmean was 596.527 cGy (RBE) (range, $125.173 \sim 974.657$ cGy [RBE]) with IMPT and $1073.826 \mathrm{cGy}$ (range, 527.924 2997.944 cGy) with IMRT, which is statistically significant $(P=0.036)$. Furthermore, the median V20 of Ipsilateral
Lung in IMPT and IMRT were $10.224 \%$ and $18.712 \%(P=0.05)$. Similarly, the median V30 of Ipsilateral Lung in IMPT and IMRT were $4.190 \%$ vs. $12.568 \%(P=0.05)$. Compared to IMRT, IMPT significantly decreases the dose to Contralateral Lung IMRT (median Dmean: 6.149 cGy vs. 34.790 cGy, $P=0.012$ ). In addition, similar dosimetric benefit was shown in the Dmean of Spinal Cord favoring IMPT (5.787 cGy vs. 138.988 cGy, $P=0.012$ ). Differences in other dosimetric parameters were not statistically significant.

\section{Discussion}

This study aims to investigate whether IMPT using PBS could spare the heart and lung better in HF-WBI compared with IMRT. Previous studies reported that proton therapy could reduce the dose to lung, heart, and subcardiac structures effectively compared with photon radiation in $\mathrm{BC}$ patients after lumpectomy or mastectomy [15-17, 20-22]. In most of the previous studies, proton therapy was performed in conventional technique by using passively scattered scanning [15-17, 22]. However, with the development of proton technology, it is necessary to conduct a dosimetric comparison between IMRT and pencil beam scanning IMPT, rather than passive scattering proton therapy (PSPT) commonly used in previous studies.

Earlier studies have demonstrated that radiotherapy can increase the risk of ischemic heart disease and thus cause excess mortality $[12,23]$. To explore the relationship between heart 
Table 2. Dosimetric data of PTV_Lumpectomy coverage.

\begin{tabular}{lcccc}
\hline & IMPT & & IMRT & P-value \\
\cline { 2 - 2 } Items & Median (range) & Median (range) & Wilcoxon Signed Rank Test \\
D99 (cGy) & $5395.608(5069.991 \sim 5709.905)$ & & $5386.749(5066.503 \sim 5708.182)$ & 0.889 \\
D98 (cGy) & $5395.914(5070.369 \sim 5710.333)$ & & $5387.214(5066.941 \sim 5708.807)$ & \\
D95 (cGy) & $5396.898(5071.439 \sim 5711.686)$ & & $5388.678(5068.129 \sim 5710.681)$ & 0.889 \\
D50 (cGy) & $5414.256(5091.207 \sim 5737.541)$ & & $5414.256(5091.207 \sim 5737.541)$ & 0.889 \\
D5 (cGy) & $5457.563(5132.946 \sim 5808.199)$ & & $5463.113(5156.500 \sim 5772.731)$ & 1.000 \\
D2 (cGy) & $5470.293(5147.111 \sim 5831.490)$ & & $5473.654(5171.760 \sim 5777.381)$ & 0.208 \\
D1 (cGy) & $5481.351(5158.820 \sim 5850.294)$ & & $5479.506(5179.703 \sim 5779.533)$ & 0.263 \\
HI (D95/D5) & $0.985(0.966 \sim 0.990)$ & & $0.989(0.981 \sim 0.993)$ & 0.093 \\
CI (V95\%/target volume) & $0.185(0.101 \sim 0.416)$ & & $0.185(0.101 \sim 0.416)$ & 0.091 \\
\hline
\end{tabular}

IMPT: intensity-modulated proton therapy, IMRT: intensity-modulated radiotherapy, D99, D98, D95, D50, D5, D2, D1: dose receiving $\geq 99 \%$, $98 \%, 95 \%, 50 \%, 5 \%, 2 \%, 1 \%$ relative volume, respectively, CI: Conformity index, HI: Homogeneity index.

Table 3. Dosimetric comparison of OARs between IMRT plans and IMPT plans.

\begin{tabular}{|c|c|c|c|c|}
\hline & & IMPT & IMRT & $P$-value \\
\hline & & Median (range) & Median (range) & Wilcoxon signed rank test \\
\hline \multirow[t]{2}{*}{ Heart } & Dmean (cGy) & $9.524(0.033 \sim 42.380)$ & $205.219(13.059 \sim 2668.997)$ & 0.012 \\
\hline & Dmax (cGy) & $952.361(0.010 \sim 4180.165)$ & $1465.509(99.437 \sim 5194.389)$ & 0.161 \\
\hline \multirow[t]{5}{*}{ Ipsilateral Lung } & Dmean (cGy) & 596.527 (125.173 974.657) & $1073.826(527.924 \sim 2997.944)$ & 0.036 \\
\hline & Dmax (cGy) & $5150.084(4465.475 \sim 5744.415)$ & $5092.091(4584.739 \sim 5297.524)$ & 0.208 \\
\hline & V5 (\%) & $31.162(6.387 \sim 51.699)$ & $48.744(17.934 \sim 98.980)$ & 0.069 \\
\hline & $\mathrm{V} 20(\%)$ & $10.224(0.589 \sim 16.911)$ & $18.712(8.740 \sim 62.213)$ & 0.050 \\
\hline & V30 (\%) & $4.190(0.247 \sim 7.170)$ & $12.568(0.058 \sim 38.278)$ & 0.050 \\
\hline \multirow[t]{5}{*}{ Contralateral Lung } & Dmean (cGy) & $6.149(0.234 \sim 38.747)$ & $34.790(8.092 \sim 494.343)$ & 0.012 \\
\hline & Dmax (cGy) & $1182.443(6.621 \sim 2632.009)$ & $409.237(92.442 \sim 4102.270)$ & 0.779 \\
\hline & V5 (\%) & $0.098(0 \sim 2.086)$ & $0.006(0 \sim 38.995)$ & 0.398 \\
\hline & $\mathrm{V} 20(\%)$ & $0.000(0.000 \sim 0.005647)$ & $0.000(0.000 \sim 1.113)$ & 0.753 \\
\hline & V30 (\%) & $0(0 \sim 0)$ & $0(0 \sim 0)$ & 0.068 \\
\hline \multirow[t]{2}{*}{ Spinal Cord } & Dmean (cGy) & $5.787(0.000 \sim 66.194)$ & $138.988(13.896 \sim 936.309)$ & 0.012 \\
\hline & Dmax (cGy) & $122.691(0.000 \sim 1276.097)$ & $506.093(36.456 \sim 2761.027)$ & 0.123 \\
\hline
\end{tabular}

Significant data is presented in bold. IMPT: intensity modulated proton therapy, IMRT: intensity modulated radiotherapy, V5, V20, V30: volume receiving $\geq 5,20,30 \mathrm{~Gy}$, respectively, Dmax: dose encompassing $2 \%$ of the volume, Dmean: mean dose.

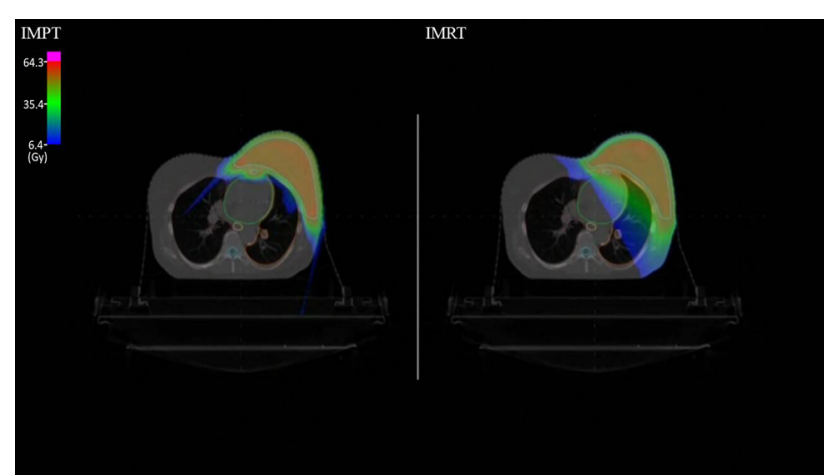

Video 1. The video shows the OARs that benefit from IMPT plans. Right side is the dose distribution of a traditional IMRT planning. Left side is of an IMPT planning for the same case. https://vcm. edpsciences.org/10.1051/vcm/2021002\#V1

exposure dose and the incidence of ischemic heart disease, Darby et al. [10] conducted a famous clinical study and found that the excess risk of ischemic heart disease caused by radiotherapy for BC patients increases linearly with an increase in the mean dose to the heart of $7.4 \%$ per Gy. Since then, we have reduced the mean dose to the heart as much as possible in our clinical work. Furthermore, Duma et al. [24] explored the increased rate of absolute radiation-induced ischemic heart disease, which found that, if a 50-year-old BC patient without cardiac risk factors exposing a mean heart dose of $3 \mathrm{~Gy}$, the risk of having at least one acute coronary event by the age of 80 years rises from 4.5 to $5.4 \%$. This risk would even rise from 8 to $9.7 \%$ with pre-existent cardiac risk factors. This risk would increase from 8 to $13.5 \%$ if the mean heart dose reached 10 Gy and in the presence of cardiac risk factors. A systematic review and meta-analysis in 2017 indicated the excess rate ratios (ERRs) of cardiac mortality was 0.04 (95\% CI, 0.02 to 0.06 ) per Gy whole-heart dose [11]. In all, in order to minimize the risk of radiation-induced ischemic heart disease, the mean heart dose should be as less as possible and preferably less than $3 \mathrm{~Gy}$. In our study, the mean heart dose closed to 0 with IMPT, much lower than the IMRT group (median Dmean 9.524 cGy vs. $205.219 \mathrm{cGy}$ ), indicating a reduction of risk of radiation-induced ischemic heart disease might be achieved by IMPT potentially.

The other non-tumor mortality was from radiation-induced lung cancer [11, 23, 25]. Grantzau et al. [14] estimated that one radiation-induced second cancer occurred in every $200 \mathrm{BC}$ 
patients treated with radiotherapy. Previous meta-analyses [11] yielded lung cancer incidence $\geq 10$ years after radiotherapy rate ratio (RR) of 2.10 (95\% CI, 1.48 to 2.98$)$, indicating 0.11 (95\% CI, 0.05 to 0.20$)$ ERR per Gy whole-lung dose. For radiationinduced lung cancer, the absolute risks from modern radiotherapy were $4 \%$ in long-term continuing smokers and $0.3 \%$ in nonsmokers. Thus, lung-sparing is critical for survival. Our results showed Dmean was significantly reduced in both the Ipsilateral (median Dmean 596.527 cGy vs. 1073.826 cGy, $P=0.036$ ) and Contralateral Lungs (median Dmean 6.149 cGy vs. $34.790 \mathrm{cGy}, P=0.012$ ) in the IMPT cohort, leading to a decrease in the excess risk of radiation-induced lung cancer. These results were consistent with previous studies. In 2014, Mast et al. [20] reported that in WBI the mean lung dose in both lungs and the Ipsilateral Lung could be reduced by IMPT significantly as compared to IMRT. In 2017, Patel et al. [17] obtained similar results in their PMRT study. Their results showed that the mean lung dose in the photon radiotherapy group was significantly higher than that in the proton therapy group, regardless of the use of passively scattered scanning or pencil beam scanning. Based on these data and studies, it can be inferred that IMPT using pencil beam scanning can effectively spare the whole lung and thus may positively impact long-term survival in breast cancer patients.

In addition to the mean lung dose, Mast et al. [20] reported that as compared to IMRT, the V5 Gy, and the V20 Gy in both lungs and in the Ipsilateral Lung could be reduced significantly in $\mathrm{BC}$ patients performed WBI in Canadian regimen. A similar dosimetric reduction was also reported in WBI and post-mastectomy radiotherapy studies $[16,17,26]$. In our study, both median V20 and V30 of the Ipsilateral Lung showed a strong tendency to reduce in IMPT, although the difference was not statistically significant $(P=0.05)$. This non-statistically significant decrease might be due to the small sample we collected.

The same reason can explain why in our study there are no differences in target coverage between the IMRT and IMPT plans, while it can be seen that IMPT can improve target coverage, conformity, and homogeneity in some other studies $[16,26]$. In addition, in terms of target coverage, the IMRT technique has been excellent. In our study, all the IMRT plans met the target coverage criteria without exception. Another reason is that our target volumes are relatively uncomplicated. We only performed a PTV_Breast to WBI and a PTV_Lumpectomy to boost. However, Bradley et al. [16] concluded that proton therapy improved target coverage for the internal mammary nodes and level 2 axilla. Meanwhile, Ares et al. [26] demonstrated that IMPT improved target coverage in complex-target irradiation without increase the integral dose. Sun et al. [21] found IMPT plans showed the best target coverage, conformity, and homogeneity in WBI of synchronous bilateral breast cancer.

The proton therapy technique used in this study was IMPT. A major advantage of pencil-beam scanning compared with passive scattering is the improved quality of the dose distribution [27]. In contrast to PSPT, IMPT can simultaneously optimize the intensities of pencil beams by using an objective function that accounts for the shape and density of targets, as well as constraints on normal tissues [28]. Thus most new institutions choose PBS as the delivery technology, and many institutions that equipped with passive scattering are upgrading their systems to PBS currently [27]. Although previous studies have not been able to verify these dosimetric advantages [17], we believe it can be further explored in future research.

In our study, DIBH technology was performed on all eight patients. Previous studies [20] showed that, unlike IMRT, the DIBH technique had no added value to IMPT. However, due to the Bragg Peak, protons are more sensitive to the effects of motion than photons. For spot scanning and IMPT, a motion was concerned potentially damaging to the homogeneity and conformity of proton plans [29]. Therefore, we believe that if available DIBH should be performed on all BC patients undergoing proton therapy.

In HF-WBI, IMPT can maximally spare the cardiopulmonary dose without compromising target coverage and may positively impact long-term survival in breast cancer patients. Considering IMPT is expensive and not widely available in China, IMPT should be performed on patients at high risk for non-tumor radiation-induced death.

\section{Conflicts of interest}

The authors have no conflicts to disclose.

\section{References}

1. van Dongen JA, Voogd AC, Fentiman IS, et al. Long-term results of a randomized trial comparing breast-conserving therapy with mastectomy: European Organization for Research and Treatment of Cancer 10801 trial. Journal of the National Cancer Institute. 2000;92(14):1143-1150.

2. Darby S, McGale P, Correa C, et al. Effect of radiotherapy after breast-conserving surgery on 10-year recurrence and 15-year breast cancer death: meta-analysis of individual patient data for 10,801 women in 17 randomised trials. Lancet (London, England). 2011;378(9804):1707-1716.

3. Bentzen SM, Agrawal RK, Aird EG, et al. The UK Standardisation of Breast Radiotherapy (START) Trial A of radiotherapy hypofractionation for treatment of early breast cancer: a randomised trial. The Lancet Oncology. 2008;9(4):331-341.

4. Bentzen SM, Agrawal RK, Aird EG, et al. The UK Standardisation of Breast Radiotherapy (START) Trial B of radiotherapy hypofractionation for treatment of early breast cancer: a randomised trial. Lancet (London, England). 2008;371 (9618):1098-1107.

5. Owen JR, Ashton A, Bliss JM, et al. Effect of radiotherapy fraction size on tumour control in patients with early-stage breast cancer after local tumour excision: long-term results of a randomised trial. The Lancet Oncology. 2006;7(6):467-471.

6. Whelan T, MacKenzie R, Julian J, et al. Randomized trial of breast irradiation schedules after lumpectomy for women with lymph node-negative breast cancer. Journal of the National Cancer Institute. 2002;94(15):1143-1150.

7. Haviland JS, Owen JR, Dewar JA, et al. The UK Standardisation of Breast Radiotherapy (START) trials of radiotherapy hypofractionation for treatment of early breast cancer: 10-year follow-up results of two randomised controlled trials. The Lancet Oncology. 2013;14(11):1086-1094.

8. Wang SL, Fang $\mathrm{H}, \mathrm{Hu} \mathrm{C}$, et al. Hypofractionated versus conventional fractionated radiotherapy after breast-conserving surgery in the modern treatment era: A multicenter, randomized 
controlled trial from China. Journal of Clinical Oncology: Official Journal of the American Society of Clinical Oncology. 2020;38(31):3604-3614.

9. Shaitelman SF, Lei X, Thompson A, et al. Three-year outcomes with hypofractionated versus conventionally fractionated wholebreast irradiation: results of a randomized, noninferiority clinical trial. Journal of Clinical Oncology: Official Journal of the American Society of Clinical Oncology. 2018;36(35):Jco1800317.

10. Darby SC, Ewertz M, McGale P, et al. Risk of ischemic heart disease in women after radiotherapy for breast cancer. The New England Journal of Medicine. 2013;368(11):987-998.

11. Taylor C, Correa C, Duane FK, et al. Estimating the risks of breast cancer radiotherapy: evidence from modern radiation doses to the lungs and heart and from previous randomized trials. Journal of Clinical Oncology: Official Journal of the American Society of Clinical Oncology. 2017;35(15):1641-1649.

12. Cuzick J, Stewart H, Rutqvist L, et al. Cause-specific mortality in long-term survivors of breast cancer who participated in trials of radiotherapy. Journal of Clinical Oncology: Official Journal of the American Society of Clinical Oncology. 1994;12(3):447-453.

13. Berrington de Gonzalez A, Curtis RE, Kry SF, et al. Proportion of second cancers attributable to radiotherapy treatment in adults: a cohort study in the US SEER cancer registries. The Lancet Oncology. 2011;12(4):353-360.

14. Grantzau T, Mellemkjær L, Overgaard J. Second primary cancers after adjuvant radiotherapy in early breast cancer patients: a national population based study under the Danish Breast Cancer Cooperative Group (DBCG). Radiotherapy and Oncology: Journal of the European Society for Therapeutic Radiology and Oncology. 2013;106(1):42-49.

15. Kammerer E, Guevelou JL, Chaikh A, et al. Proton therapy for locally advanced breast cancer: A systematic review of the literature. Cancer Treatment Reviews. 2018;63:19-27.

16. Bradley JA, Dagan R, Ho MW, et al. Initial report of a prospective dosimetric and clinical feasibility trial demonstrates the potential of protons to increase the therapeutic ratio in breast cancer compared with photons. International Journal of Radiation Oncology, Biology, Physics. 2016;95(1):411-421.

17. Patel SA, Lu HM, Nyamwanda JA, et al. Postmastectomy radiation therapy technique and cardiopulmonary sparing: A dosimetric comparative analysis between photons and protons with free breathing versus deep inspiration breath hold. Practical Radiation Oncology. 2017;7(6):e377-e384.

18. RTOG. Breast Cancer Atlas for Radiation Therapy Planning: Consensus Definitions. 2017.
19. Hodapp N. Prescribing, recording, and reporting photon-beam Intensity-Modulated Radiation Therapy (IMRT). Journal of the ICRU. 2010;10(1):NP.

20. Mast ME, Vredeveld EJ, Credoe HM, et al. Whole breast proton irradiation for maximal reduction of heart dose in breast cancer patients. Breast Cancer Research and Treatment. 2014;148 (1):33-39.

21. Sun T, Lin X, Tong Y, et al. Heart and cardiac substructure dose sparing in synchronous bilateral breast radiotherapy: a dosimetric study of proton and photon radiation therapy. Frontiers in Oncology. 2019;9:1456.

22. MacDonald SM, Patel SA, Hickey S, et al. Proton therapy for breast cancer after mastectomy: early outcomes of a prospective clinical trial. International Journal of Radiation Oncology, Biology, Physics. 2013;86(3):484-490.

23. Clarke M, Collins R, Darby S, et al. Effects of radiotherapy and of differences in the extent of surgery for early breast cancer on local recurrence and 15-year survival: an overview of the randomised trials. Lancet (London, England). 2005;366 (9503):2087-2106.

24. Duma MN, Molls M, Trott KR. From heart to heart for breast cancer patients - cardiovascular toxicities in breast cancer radiotherapy. Strahlentherapie und Onkologie : Organ der Deutschen Rontgengesellschaft [et al]. 2014;190(1):5-7.

25. Wu GX, Nelson RA, Kim JY, et al. Non-small cell lung cancer as a second primary among patients with previous malignancy: Who is at risk? Clinical Lung Cancer. 2017;18(5):543-550.e3.

26. Ares C, Khan S, Macartain AM, et al. Postoperative proton radiotherapy for localized and locoregional breast cancer: Potential for clinically relevant improvements? International Journal of Radiation Oncology, Biology, Physics. 2010;76 (3):685-697.

27. Depuydt T. Proton therapy technology evolution in the clinic: impact on radiation protection. Annals of the ICRP. 2018;47(34):177-186.

28. Chang JY, Zhang X, Knopf A, et al. Consensus Guidelines for implementing pencil-beam scanning proton therapy for thoracic malignancies on behalf of the PTCOG thoracic and lymphoma subcommittee. International Journal of Radiation Oncology, Biology, Physics. 2017;99(1):41-50.

29. Gueulette J, Blattmann H, Pedroni E, et al. Relative biologic effectiveness determination in mouse intestine for scanning proton beam at Paul Scherrer Institute, Switzerland. Influence of motion. International Journal of Radiation Oncology, Biology, Physics. 2005;62(3):838-845.

Cite this article as: Lin R, Shan J, Yuan T, Qian C. Dosimetric comparison of intensity-modulated proton radiotherapy versus intensitymodulated photon-based radiotherapy for breast cancer. Visualized Cancer Medicine. 2020; $2,5$. 